\title{
Performance Analysis of ZigBee Protocol in the Large- Scale Network Topologies
}

\author{
Gürcan Cetin \\ Dep't of Information Systems \\ Engineering, \\ Muğla Sıtkı Koçman University
}

\author{
Hüseyin Gürüler \\ Dep't of Information Systems \\ Engineering, \\ Muğla Sıtkı Koçman University
}

\author{
Mehmet Osman Devrim \\ Dep't of Information Systems \\ Engineering, \\ Muğla Sıtkı Koçman University
}

\author{
Sefa Tuncay \\ Dep't of Information Systems Engineering, \\ Muğla Sıtkı Koçman University
}

\begin{abstract}
Wireless Sensor Networks (WSN) are self-configurable adhoc networks. To set up a seamlessly interoperable a WSN, a wireless sensor device technology called ZigBee is used. Moreover, ZigBee is an IEEE 802.15.4 based specification for a group of high-level communication protocols used to build area networks having both small range and wide range coverage. The design of the communication infrastructure and hardware components are crucial in the large-scale ZigBee networks in order to ensure communication efficiency in the real world applications. This study presents a performance analysis of a large-scale ZigBee networks for three different topologies comparatively. The performance of ZigBee devices has been analyzed in the network simulator OPNET in terms of total end-to-end delay, MAC throughput and MAC load.
\end{abstract}

\section{Keywords}

Large-scale WSN, performance analysis topologies, ZigBee

\section{INTRODUCTION}

Recent advances in computer, communication and microelectronic technology have revolutionized WSN research. The use of WNSs has expanded with the reduction of the size of sensors and the decreasing of their prices [1]. So, electromechanical WSN ecosystems that can be monitored or controlled by the Internet of Things (IoT) components have emerged. These ecosystems consist of uniquely identified devices which are capable of Device-to-Device (D2D) communication.

D2D communication is usually carried out by short-range communication protocols via multi-hop transmissions instead of using the Internet infrastructure. Digital Enhanced Cordless Telecommunications Ultra Low Energy (DECT ULE), Bluetooth Low Energy (BLE), Radio Frequency Identification (RFID) and Near Field Communication (NFC) are protocols developed for this purpose [2]. Moreover, IEEE 802.15.4 standard also focuses on short-range wireless links (WPANs Wireless Personal Area Networks) with low data rate (0.01$250 \mathrm{Kbits} / \mathrm{s})$ and low complexity. It supports ZigBee, IPv6 over Low power WPAN (6LoWPAN) and Wireless Highway Addressable Remote Transducer Protocol (WirelessHART).

ZigBee based on IEEE 802.15.4 standard is generally considered and chosen to build WPAN areas due to its long battery life, low cost, low data rate, reliability and flexible structure. In the ZigBee protocol, IEEE is responsible to development of physical layer (PHY), media access control
(MAC) layer and data link layer, while ZigBee Alliance is responsible to data transmission encryptions, determining logic networks, application interface specifications and communication specifications between system products [3]. ZigBee protocol stack can be seen in Figure 1 [4].

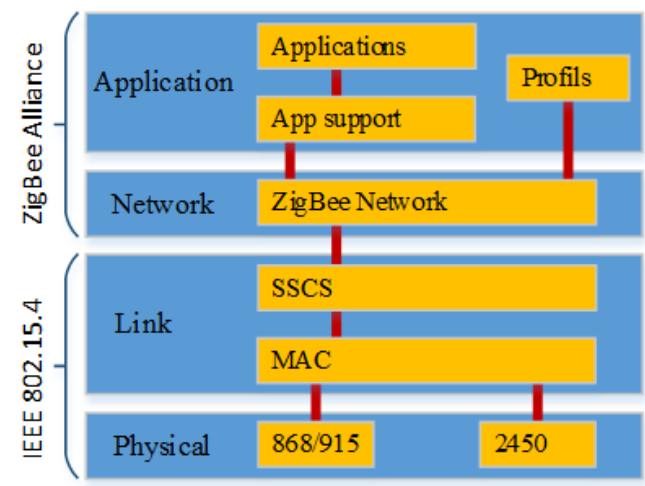

Fig 1: IEEE 802.15.4 / ZigBee protocol stack

With ZigBee technology, it is possible to build complex WSN infrastructures, to expand them, and to communicate with other networks [5]. Considering the studies have been done in recent years, ZigBee have been used data acquisition systems [3], environmental monitoring [6], automated control mechanisms [7], agriculture [8], wearable healthcare applications [9], office and home automation systems [10] [11]. As can be seen from these studies, ZigBee nodes can wirelessly communicate with each other in their coverage areas, sense their environments and perform common tasks together. However, the design of the communication infrastructure and hardware components are crucial in the large-scale ZigBee networks in order to guarantee communication efficiency. For this purpose, different studies were conducted by the researchers to analyze the performance of the existing protocol. The study [12] focused on real-time capabilities and reliability of ZigBee, and outlined which degree the standard accomplish real time requirements. The researches considered data delivery delay, goodput, throughput and packet error rate parameters. In [13], researchers demonstrated the performance of ZigBee through two new geometric (random and octagonal) structures in large-scale networks. The results showed that octagonal structure better performance than random structure in terms of end-to-end delay, MAC load and MAC delay. In the another work [14], ZigBee was analyzed for large-scale WSN 
applications. The performance was evaluated with power consumption and goodput parameters.

In this study, the large-scale network performance of ZigBee technology over star, cluster tree and mesh topologies have been analyzed using the OPNET network simulation tool. The main focus performed analysis was on MAC throughput, MAC load and end-to-end delay parameters. It is expected that the performance results will guide the design of a true large-scale ZigBee network application.

The paper is organized as follows. Section 2 gives an overview of the ZigBee technology, its device types and topologies. The simulation parameters and the results are given in Section 3. Finally, Section 4 concludes this paper.

\section{ZIGBEE}

ZigBee technology based on IEEE 802.15.4 is used to set up WPAN areas with low data transmission rate in short distance communication [3]. IEEE 802.15.4 supports two frequency bands; 868/915 MHz low-band and $2.4 \mathrm{GHz}$ high-band. It uses Binary Phase Shift Keying (BPSK) modulation in lowband frequencies, whereas Offset Quadrature Phase Shift Keying (OQPSK) modulation is used for high-band frequencies [12]. The main reason of using high-band in ZigBee applications is that the number of channels is high compared to other available frequencies as shown in Table 1 [15]. Thus, in large-scale WSN applications, the frame transmission time and therefore the energy consumed for each transmitted and received bit is reduced [3].

Table 1. IEEE 802.15.4 frequency bands [14]

\begin{tabular}{|l|l|l|l|}
\hline Band & $\mathbf{8 6 8} \mathbf{~ M H z}$ & $\mathbf{9 1 5} \mathbf{~ M H z}$ & $\mathbf{2 . 4} \mathbf{~ G H z}$ \\
\hline Region & EU, Japan & US & Worldwide \\
\hline Channels & 1 & 10 & 16 \\
\hline Data Rate & $20 \mathrm{kbps}$ & $40 \mathrm{kbps}$ & $250 \mathrm{kbps}$ \\
\hline
\end{tabular}

ZigBee protocol uses a talk-when-ready collision prevention mechanism. According to this procedure, a data is sent when necessary, and it is confirmed with an acknowledgement message by receiver. If an acknowledgement message is not received in response, a collision occurs and the data packet is retransmitted. This significantly increases the reliability of the system. Moreover, the main features of the ZigBee are [3] [16];

- Low power consumption (with 2 AA batteries, 6 - 24 months depending on the application)

- Low data rate (max 250Kbps)

- Low cost

- Supports 65535 nodes in a single network.

- Uses smaller packets compared to Wi-Fi and Bluetooth

- Uses 16-bit short structure or 64-bit MAC addressing mechanism.

- The coverage area varies from $100 \mathrm{~m}$ (indoor) to $1.5 \mathrm{~km}$ (outdoor) depending on the transmission power or environmental factors.

- Provides acknowledge data transmission and optional beacon structure.

- Supports energy detection (ED), Link Quality
Indication (LQI) and Multilevel security.

\subsection{ZigBee Network Devices}

IEEE 802.15.4 defines two types device capabilities in terms of functionality; full-function device (FFD) and reducedfunction device (RFD). FFDs such as ZigBee Coordinator (ZC) and ZigBee Router (ZR) are using for making to intelligent node requirements on platforms having all MAC services [11]. On the other hand, RFDs such as ZigBee End Device (ZED) are preferred to meet low performance requirements in low-cost, low energy consumption and easyto-use platforms [2]

A ZigBee network is initiated and managed by a ZC. It controls devices joined to the network or disconnected from the network. It also has a neighbor table of devices that have a neighborhood. For this reason, $\mathrm{ZC}$ requires more memory and processing power than other devices. ZC is also working as a gateway to other networks. ZCs collaborate with each other to perform data routing and self-organization operations. ZRs relay traffic for ZEDs which do not have data routing capability, and they are used to expand the coverage of wireless sensor network.

\subsection{Topology of ZigBee Wireless Networks}

ZigBee protocols supports multiple network topologies like centralized star, cluster-tree and mesh. The number of ZC, ZR and ZED is set according to application design.

\subsubsection{Star Topology}

Star Topology uses a Multipoint-to-Point connectivity between nodes where there is a ZC in the center. There is a single hop for all connections, and all messages are transmitted over the ZC. Figure 2 shows test network structure for star topology that has a ZC in the center and the ZEDs on the edges. Star topology is attractive due to its simple configuration. However, there are some disadvantages. When a ZC fails, all network traffic is interrupted because all traffic travel passed through the ZC. Another disadvantage of this situation is that the ZC can easily become a bottleneck, especially in a large scale network with a high number node [17].

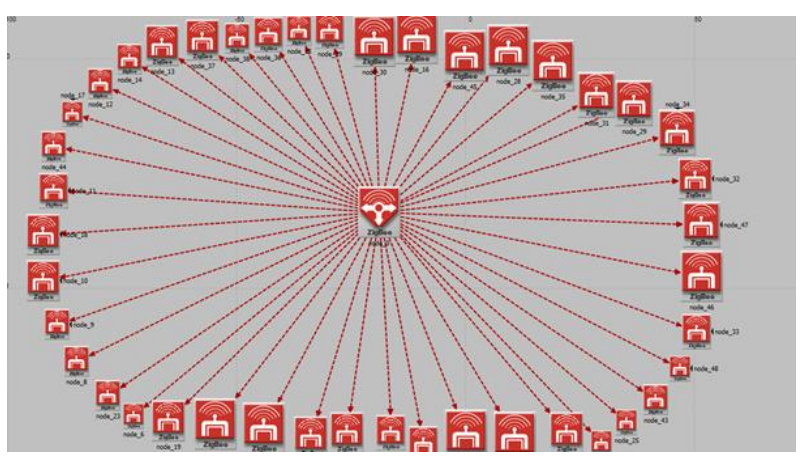

Fig 2: Star topology for ZigBee protocol

\subsubsection{Tree Topology}

In the tree topology, devices communicate with each other at a hierarchical level. There is a $\mathrm{ZC}$ on the top of the tree structure, and it is connected to ZRs at lower levels. The ZEDs and ZRs at lowest levels [18]. Figure 3 shows test network structure for tree topology.

In order to send data packets to destination nodes in the tree topology, the source node must pass these packets to its parent node. Parent node is a higher up one level of the source node in the topology, and the packets are continuously transmitted 
the parent nodes until the source node is reached. The disadvantage of this topology is that the number of potential routes for a message is only one. If a ZR fails, all children nodes of that ZR is disconnected from the rest of the network [17].

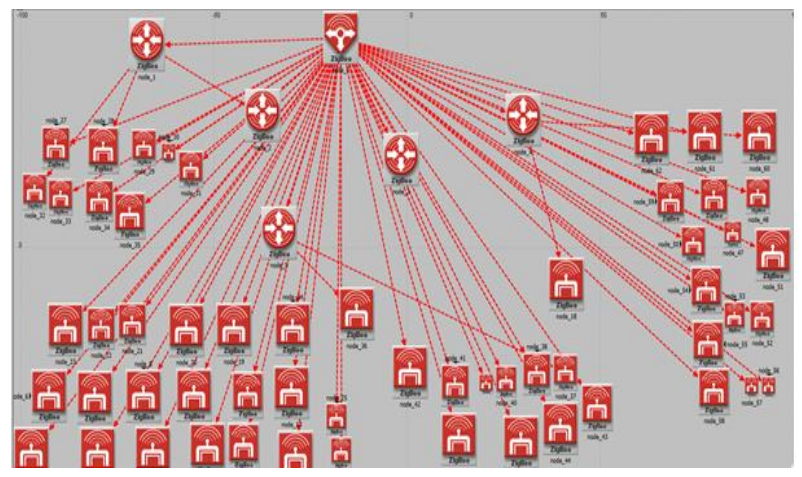

Fig 3: Tree topology for ZigBee protocol

\subsubsection{Mesh Topology}

In this topology, all devices communicate with each other using the AODV (Ad-hoc Ondemand Distance Vector Routing) algorithm. If the route to be transmitted is not known, the source device broadcasts a route request packet to entire network via AODV algorithm in order to find out where and in which direction the destination device in located. This packet contains the packet sequence number, the metric information, the destination network address and source network address fields. The metric is a cost values calculated through the manufacturer and various standards by using parameters when finding the most advantageous route of communicating between the devices. The devices receiving the route request packet from source device only transmit the route information of the destination device with the metric. When a response is received from more than one device to the route request packet, the data packet is sent from the path with the lowest metric cost [19]. Figure 4 shows test network structure for mesh topology.

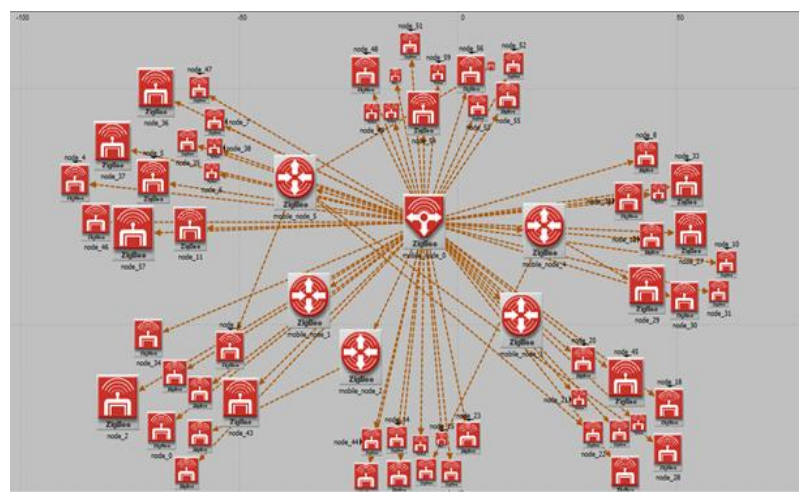

Fig 4: Mesh topology for ZigBee protocol

\section{SIMULATION AND RESULTS}

In this section, we are interested in a large scale ZigBee network environment with star, tree and mesh topologies separately. The star, tree and mesh network structures evaluated in the analysis are given in Figure 2, Figure 3 and Figure 4 respectively. All nodes in the simulation randomly distributed according to Random structure model. All network topologies in the simulation infrastructures have almost equal number of ZED, ZR and ZC. The Riverbed OPNET (Optimized Network Engineering Tool) simulator has been used to configure all ZigBee network parameters and to evaluate their performance on the large scale networks [20]. Transmission band, traffic packet size, transmit power and other simulation parameters have set to all scenarios that shown in Table 2.

Table 2. ZigBee network simulation parameters

\begin{tabular}{|l|l|}
\hline Parameters & Value \\
\hline ACK Status & Disabled \\
\hline Number of Retransmissions & 5 \\
\hline Channel Sensing Duration & 0.1 \\
\hline Data Rate & Auto Calculate \\
\hline Transmission Band & $2450 \mathrm{MHz}$ \\
\hline Transmit Power & 0.05 \\
\hline Number of nodes in Mesh topology & 55 \\
\hline Number of nodes in Tree topology & 57 \\
\hline Number of nodes in Star topology & 48 \\
\hline No. of ZigBee Routers (Mesh, Tree) & 5 \\
\hline Max number of ZigBee Coordinator & 1 \\
\hline Maximum Depth & 5 \\
\hline Traffic Destination & Random \\
\hline Traffic Interarrival Time & Constant (1.0) \\
\hline Traffic Packet Size & Constant (1024) \\
\hline Start time & Uniform (20,21) \\
\hline Network Dimensions & $500 \mathrm{~m} x$ 500m \\
\hline
\end{tabular}

\subsection{End to End Delay}

End-to-end delay refers to the total of time required for a packet to be transmitted across a network from a source to the destination. It depends on the number of routers between the source and the destination, and it is a crucial metric to evaluate the network performance. End-to-End delay can be expressed as equation (1), if the network is optimal.

$$
D_{\text {end-end }}=N \times\left(d_{\text {proc }}+d_{\text {trans }}+d_{\text {prop }}\right)
$$

Where;

$$
\begin{aligned}
& \mathrm{N}=\text { Number of Routers }+1 ; \\
& \text { d proc = Processing Delay; } \\
& \text { dtrans = Transmission Delay; } \\
& \text { d prop = Propagation Delay; }
\end{aligned}
$$

Since each router has its own dproc, dtrans and dprop values at heterogeneous links, the formula given equation (1) is applied to homogenous network structures. 


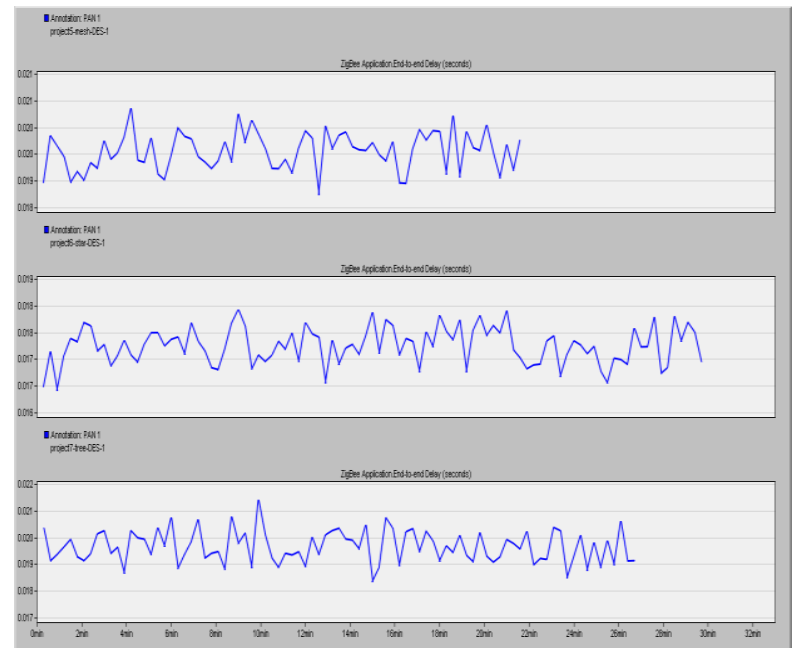

Fig 5: End to End delay for ZigBee Mesh, Star and Tree Topologies

According to the end-to-end delay analysis results shown in Figure 5; Since there are an equal number of routers used in the mesh and tree topology, the end-to-end delay are higher than the star topology. The main reason of that there is no ZR in the star topologies, and the packets hop only one node to reach ZC. The highest peak in the simulation process occurred in the Tree topology.

\subsection{Throughput}

The MAC layer throughput refers to the total number of bits transmitted by the ZigBee MAC layer towards higher layers in all nodes [13]. The throughput rate of each node is calculated by dividing the total number of successfully received bits to runtime. It can be seen in equation (2).

$$
\text { Throughput }=\frac{\text { the total number of bits }}{\text { runtime }}
$$

Figure 6 shows the MAC layer throughput values for $\mathrm{ZC}$ in the mesh, star and tree network topologies. According to the Figure 6 , the highest throughput value has been obtained in the mesh topology, while the least throughput value has been in the star topology. This is mainly due to the fact that the mesh topology has a high data transmission rate. In other words, the topology has the least data loss.

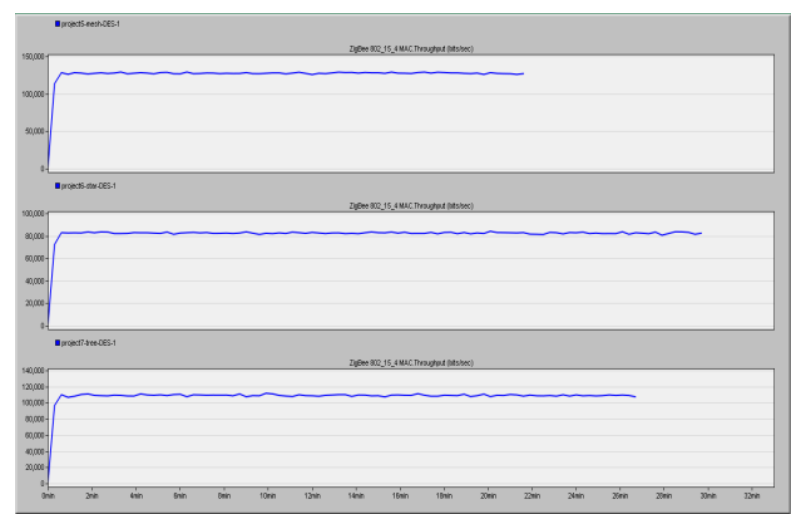

Fig 6: Throughput for ZigBee Mesh, Star and Tree Topologies

\subsection{Load}

The MAC layer load refers to the total load (bits/sec) submitted by the ZigBee MAC from higher layers in all nodes [13]. Figure 6 shows the MAC load values for $\mathrm{ZC}$ in the mesh, star and tree network topologies separately. According to the Figure 7, the highest value of gained load has been obtained in the mesh topology, while the least load value has been in the star topology. The main reason of that the data transmission is maximum in the mesh topology. ZC cope up with all data requests from its queue. In this model, randomly a lot of requests come in the $\mathrm{ZC}$ queue from the different nodes in the WSN infrastructure.

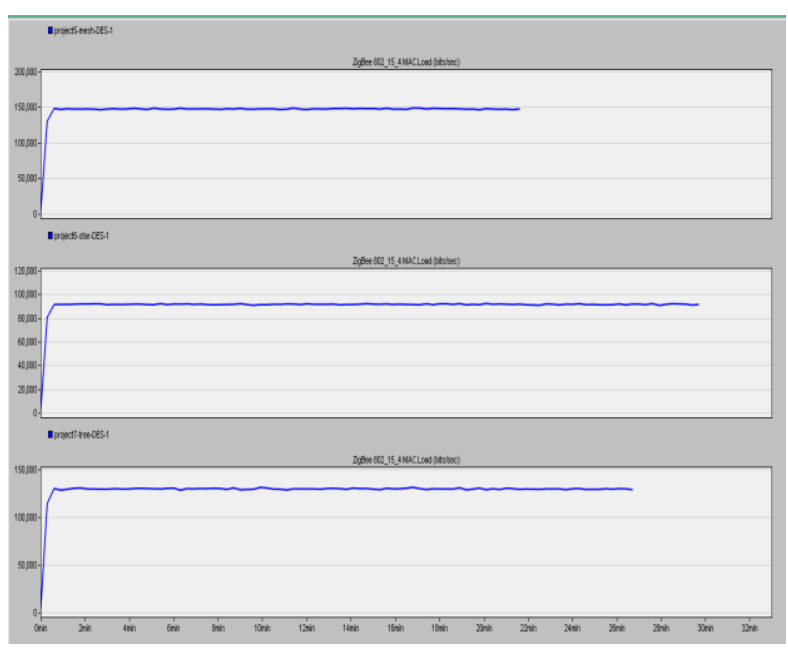

Fig 7: Load for ZigBee Mesh, Star and Tree Topologies

\section{CONCLUSION}

In this study, three different scenarios were simulated and analyzed to examine WSN topological performances in the large scale network. We have compared Star, Mesh, and Tree network topologies with each other and examined some critical performance statistics such as end-to-end delay, MAC throughput and MAC load. According to the study results;

The star topology supporting the single-hop is most suitable for delay critical applications. The results evaluation confirms that the performances of mesh topology was the best according to all performance factors. Therefore, mesh topology is preferable in the design of WSN considering higher throughput and reliable message transmission. On the other hand, tree topology gives average results when compared with the other topologies.

\section{REFERENCES}

[1] Merrett, G. V., and Tan, Y. K., 2010. Wireless Sensor Networks: Application - Centric Design, InTech.

[2] Bello, O., Zeadally, S., and Badra, M., 2017. Network layer inter-operation of Device-to-Device communication technologies in Internet of Things (IoT), Ad Hoc Networks, No 57, 52-62.

[3] Shariff, F., Rahim, N. A., and Ping, H. W., 2015. ZigbeeBased Data Acquisition System for Online Monitoring of Grid-connected Photovoltaic System, Expert Systems with Applications, No 42, 1730-1742.

[4] Çetin, G., Okul, F., and Karadaş, S., 2017. A wireless Sensor Network Application for Vehicle Tracking in Campus Areas, Mugla Journal of Science and Technology, No 2(3), 150-154.

[5] Masica, K., 2007. Recommended Practices Guide for Securing ZigBee Wireless Networks in Process Control System Environments Draft, Lawrence Livermore National Laboratory.

[6] Chengbo, Y., Yanzhe, C., Lian, Z., and Shuqiang, Y., 2009. ZigBee Wireless Sensor Network in Environmental Monitoring Applications, 5th IC on 
Wireless Communications, Networking and Mobile Computing.

[7] Barankova, I., Mikhailova, U. and Lukianov, G., 2016. Automated Control System of a Factory Railway Transport Based on ZigBee, 2nd IC on Industrial Engineering, Applications and Manufacturing.

[8] Rodiriquez, R. Y., Julcapoma, M. R., and Jacinto, R. A., 2016. Network Monitoring Environmental Quality in Agriculture and Pisciculture with Low Power Sensor Nodes Based On ZigBee and GPRS Technology, XXIII IC on Electronics, Electrical Engineering and Computing.

[9] Lee, C. C., Shen, Y., Lee, W. C., Hung, F. H., and Tsang, K. F., 2016. ZigBee LNA Design for Wearable Healthcare Application, 14th International Conference on Industrial Informatics.

[10] Huang, P. C., 2009. ZigBee Wireless Network Application Research Case Study within Taiwan University Campus, Proceeding of the 8th IC on Machine Learning and Cybernetics.

[11] Lin, S., Liu, J., and Fang, Y., 2007. ZigBee based wireless sensor networks and its applications in industrial, IEEE IC on Automation and Logistics.

[12] Chaari, L., and Kamoun, L., 2011. Performance Analysis of IEEE 802.15.4/ZigBee Standard Under Real Time Constraints, International Journal of Computer Networks \& Communications, No 5(3), 235-250.

[13] Biddut, J. H., Islam, N., Jany, M. H. R. and Swapna, A. I., 2015. Performans Analysis of Large Scale ZigBee
Network Design Through Geometric Structure, 2nd IC on Electrical Information and Communication Technology.

[14] Kohvakka, M., Kuorilehto, M., Hannikainen, M., and Hamalainen, T. D., 2006. Performance Analysis of IEEE 802.15.4 and ZigBee for Large Scale Wireless Sensor Network Applications, International Symposium on Performance Evaluation of Wireless Ad Hoc, Sensor, and Ubiquitous Networks.

[15] Silveria, E., and Bonho, S., 2016. Temperature Monitoring Through Wireless Sensor Networks Using An 802.15.4/802.11 Gateway, IFAC-PapersOnLine, No 30(49), 120-125.

[16] Ajgaonkar, P., 2010. Simulation Studies on ZigBee Communications for Home Automation and Networking, The University of Toledo M.Sc.Thesis, Toledo.

[17] Hammoshi, M., Al-Ani, R., Sayed, A., and Alshihi, M., 2014. Managing Different ZigBee Network Topologies Using OPNET, 2nd IC on Applied Information and Communications Technology.

[18] "IEEE, IEEE Wireless Standarts Zone", http://standards. ieee.org/wireless/overwiev.html, 25 June 2017. [Online]

[19] Farahani, S., 2008. ZigBee Wireless Networks and Transceivers, Elseiver.

[20] "Riverbed, Steel Central Riverbed Modeler | Riverbed GB”, 26 June 2018, https://www.riverbed.com/gb/ products/steelcentral/steelcentral-riverbed-modeler.html [Online]. 